\title{
High resolution magnetic resonance imaging in pathogenesis diagnosis of single lenticulostriate infarction with nonstenotic middle cerebral artery, a retrospective study
}

Li-Li Sun ${ }^{1}$, Zhong-Hao Li', Wen-Xiong Tang', Lei Liu', Fei-Yan Chang ${ }^{2}$, Xue-Bin Zhang ${ }^{2}$, Wei-Jie Ye ${ }^{1}$, Shuo Lu', Zun-Jing Liu ${ }^{1 *}$ (D) and Xian-Jin Zhu ${ }^{2^{*}}$

\begin{abstract}
Background: It is usually difficult to identify stroke pathogenesis for single lenticulostriate infarction with nonstenotic middle cerebral artery (MCA). Our aim is to differentiate the two pathogeneses, non-branch atheromatous small vessel disease and branch atheromatous disease (BAD) by high-resolution magnetic resonance imaging (HR-MRI).

Methods: Thirty-two single lenticulostriate infarction patients with nonstenotic MCA admitted to the China-Japan Friendship Hospital from December 2014 to August 2017 were enrolled for retrospective analysis. National Institutes of Health Stroke Scale (NIHSS), modified Rankin Scale (mRS), atherosclerotic risk factors, imaging features, and the characteristic of MCA vessel wall in HR-MRI were evaluated.

Results: MCA plaques were detected in 15(46.9\%) patients which implied BAD and 8 of 15 (53.3\%) patients had plaques location in upper dorsal side of the vessel wall. Patients with HR-MRI identified plaques had a significantly larger infarction lesion length $(1.95 \pm 0.86 \mathrm{~cm}$ versus $1.38 \pm 0.55 \mathrm{~cm} ; P=0.031)$ and larger lesion volume $\left(2.95 \pm 3.94 \mathrm{~cm}^{3}\right.$ versus $0.90 \pm 0.94 \mathrm{~cm}^{3} ; P=0.027$ ) than patients without plaques. Patients with HR-MRI identified plaques had a significant higher percentage of proximal lesions than patients without plaques $(P=0.055)$. However, according to the location of MCA plaques, there were no significant differences in terms of imaging features, NIHSS and mRS.
\end{abstract}

Conclusion: We demonstrated high frequency of MCA atheromatous plaques visualized in single lenticulostriate infarction patients with nonstenotic MCA by using HR-MRI. Patients with HR-MRI identified plaque presented larger infarction lesions and more proximal lesions than patients without plaque, which were consistent with imaging features of BAD. HR-MRI is an important and effective tool for identifying stroke etiology in patients with nonstenotic MCA.

Keywords: Lenticulostriate infarction, Branch atheromatous disease, Small vessel disease, High-resolution magnetic resonance imaging, Pathogenesis

\footnotetext{
* Correspondence: liuzunjing@163.com; xianjinzhu@yeah.net

${ }^{1}$ Department of Neurology, China-Japan Friendship Hospital, 2 Yinghua

Dongjie, Hepingli, Beijing 100029, China

2Department of Radiology, China-Japan Friendship Hospital, 2 Yinghua

Dongjie, Hepingli, Beijing 100029, China
}

(c) The Author(s). 2018 Open Access This article is distributed under the terms of the Creative Commons Attribution 4.0 International License (http://creativecommons.org/licenses/by/4.0/), which permits unrestricted use, distribution, and reproduction in any medium, provided you give appropriate credit to the original author(s) and the source, provide a link to the Creative Commons license, and indicate if changes were made. The Creative Commons Public Domain Dedication waiver (http://creativecommons.org/publicdomain/zero/1.0/) applies to the data made available in this article, unless otherwise stated. 


\section{Background}

Stroke is an important public health problem throughout the world with major mortality and severe morbidity. The accurate identification of stroke etiology is important for guiding patient management and prognosis [1]. Lenticulostriate infarction is ischemia in the territory supplied by the deep perforating branches of the middle cerebral artery (MCA). Lenticulostriate arteries supply the basal ganglia and most of the internal capsule [2]. There are two different vascular pathogenesis in single lenticulostriate infarction with nonstenotic MCA: 1) branch atheromatous disease (BAD), atheromatous plaque of MCA at the orifice of lenticulostriate arteries and 2) non-branch atheromatous small vessel disease, histologically characterized by lipohyalinotic degeneration of lenticulostriate arteries themselves [3]. BAD was proposed by Caplan in 1989 [4]. It was identified as a new pathogenesis caused by an occlusion or stenosis at the origin of a deep penetrating artery of the brain. Nonbranch atheromatous small vessel disease is different from BAD showed lower mortality and higher risk of death when with anticoagulant therapy [1].

However, it is usually difficult to distinguish BAD from non-branch atheromatous small vessel disease for practicing clinicians. Conventional imaging techniques, magnetic resonance angiography (MRA), computed tomography angiogram (CTA), and digital subtraction angiography (DSA) can't identify the two pathogenesis in nonstenotic arteries because those only show the information of the lumen, not the vessel wall.

High-resolution magnetic resonance imaging (HR-MRI) could display the features of arterial wall, and recent studies showed that it was also an effective tool for identifying plaque of intracranial arteries [5-7]. In this study, we aimed to determine whether HR-MRI can identify early stage atherosclerotic plaque in patients with nonstenotic MCA on MRA, and investigate the clinical distinction of the two pathogenesis BAD and non-branch atheromatous small vessel disease.

\section{Methods}

\section{Patients}

Between December 2014 and August 2017, patients from China-Japan Friendship Hospital met the following criteria were enrolled: (1) a single new-onset lenticulostriate artery territory infarction on diffusion weighted imaging (DWI); and (2) no relevant MCA disease on MRA. Patients with the following conditions were excluded: (1) had a definite cardioembolic source (e.g., atrial fibrillation, recent myocardial infarction, dilated cardiomyopathy, valvular heart disease, and infectious endocarditis); (2) stenosis of ipsilateral internal carotid artery; or (3) nonatherosclerotic vasculopathy (e.g., dissection, arteritis, and moyamoya disease).
For all enrolled patients, age, gender, atherosclerotic risk factors including hypertension (defined as receiving medication for hypertension or blood pressure $>140$ / $90 \mathrm{mmHg}$ on repeated measurements), diabetes mellitus (defined as receiving medication for diabetes mellitus, fasting blood glucose $\geqq 126 \mathrm{mg} / \mathrm{dL}$, and/or 2-h postprandial blood glucose $\geqq 200 \mathrm{mg} / \mathrm{dL}$ ), hyperlipidemia (defined as receiving cholesterol reducing agents or overnight fasting cholesterol level $>200 \mathrm{mg} / \mathrm{dL}$ ), hyperhomocysteinemia (defined as serum level of total homocysteine $\geq 15 \mu \mathrm{mol} / \mathrm{L}$ ), tobacco consumption, and history of stroke and coronary heart disease were collected. National Institutes of Health Stroke Scale (NIHSS) score was measured at the time of admission and discharge. Modified Rankin Scale (mRS) score was measured at the time of discharge. All patients underwent $24 \mathrm{~h}$ of electrocardiographic monitoring and transthoracic echocardiography to exclude silent cardioembolism.

\section{Imaging protocol}

Brain MRI examinations were performed in a $3 \mathrm{~T}$ scanner (Ingenia; Philips Healthcare, Best, the Nederland) with a 15-channel phased-array head coil. Axial T2-weighted, fluid-attenuated inversion recovery and DWI were obtained to evaluate infarction lesions. All above sequences had $5 \mathrm{~mm}$ slice thickness and $1.0 \mathrm{~mm}$ interstice gap. 3D time-of-flight (TOF) MRA was obtained with the following parameters: repetition time/echo time $=21 \mathrm{~ms} / 3.2 \mathrm{~ms}$, field of view $(\mathrm{FOV})=200 \times 200 \times 344 \mathrm{~mm} 3$, matrix $=$ $400 \times 287 \times 287$, and number of signal averages $($ NSA $)=1$. Acquisition $(\mathrm{ACQ})$ voxel volume was $0.5 \times 0.7 \times 1.2 \mathrm{~mm} 3$. Reconstruction (REC) voxel volume was $0.5 \times 0.5 \times 0$. $6 \mathrm{~mm} 3$. 3D volumetric isotropic turbo spin echo acquisition (VISTA) images were obtained with the following parameters: repetition time/echo time $=1300 \mathrm{~ms} / 36 \mathrm{~ms}$, FOV $=140 \times 200 \times 135 \mathrm{~mm} 3$, matrix $=280 \times 332 \times 270$, $\mathrm{NSA}=2$. ACQ voxel volume was $0.5 \times 0.6 \times 0.5 \mathrm{~mm} 3$. REC voxel volume was $0.5 \times 0.5 \times 0.5 \mathrm{~mm} 3$. The short axial cross-sections were constructed automatically with $0.5 \mathrm{~mm}$ slice thickness. MR images were transferred to a digital picture archiving and communication (PACS) workstation. MCA plaques were assessed on both short axial and long axial images. The location of plaques and the relationship between plaques and infarcts were evaluated carefully.

Based on fluid-attenuated inversion recovery imaging, leukoaraiosis was graded from 1 to 3 according to the visual Fazekas scale:grade 1, mild (single lesions $<10 \mathrm{~mm}$; areas of "grouped" lesions $<20 \mathrm{~mm}$ in any diameter); grade 2 , moderate (single hyperintense lesions between 10 to $20 \mathrm{~mm}$; areas of "grouped" lesions $\geq 20 \mathrm{~mm}$ in any diameter; no more than "connecting bridges" between individual lesions); and grade 3, severe (single lesions or confluent areas of hyperintensity $\geq 20 \mathrm{~mm}$ in any diameter) [8]. Fazekas scale 
of deep white matter changes with scores of 2 and 3 considered significant leukoaraiosis.

Based on DWI, the largest lesion was used to determine the volume of infarction: $1 / 2 \times$ diameter of length $\times$ diameter of width $\times(0.5 \times$ numbers of DWI slices of acute infarction). Lesion location in relation to the parent artery (MCA) was dichotomized as proximal infarction lesion (extending to the basal surface of the parent artery) and distal infarction lesion (not extending to the basal surface of the parent artery; Fig. 1) [9].

\section{Statistical analysis}

Continuous values were expressed as mean \pm SD and nominal variables as count and percentages. Median values and the interquartile range (IQR) were computed for nonnormally distributed variables. The Fishers exact test or $\chi^{2}$ test was performed to assess the categorical variables. The normally distributed continuous variables were analyzed by independent sample $t$-test. Continuous variables nonnormally distributed were analyzed by Mann-Whitney U-test. Statistical analyses were conducted using SPSS for Windows (version 20.0). A 2-sided value of $P<0.05$ was considered to indicate a statistically significant difference.

\section{Results}

\section{General patient characteristics}

During the study period, a total of 32 patients (10 female and 22 male) were enrolled. The mean age was $60.97 \pm$ 11.54. Atherosclerotic risk factors included hypertension in 22 patients $(69.0 \%)$, diabetes mellitus in 8 patients (25\%), hyperlipidemia in 20 patients (62.5\%), hyperhomocysteinemia in 7 patients $(21.9 \%)$, and tobacco consumption in 10 patients (31.3\%). 3 patients (9.4\%) had histories of stroke and 0 patient had histories of coronary heart disease. The median NIHSS score at admission was 2.5 (IQR: 1.0 to 4.0). The median NIHSS score at discharge was 1.5 (IQR: 1.0 to 3.75). The median mRS score at discharge was 1.0 (IQR: 0 to 2.0).

MCA plaques were detected in $15(46.9 \%)$ patients and, of these, 8 (53.3\%) patients had plaques' location involved upper dorsally part of the vessel wall (Table 1). The mean infarction lesion length was $1.64 \pm 0.76 \mathrm{~cm}$. The median number of infarction lesion slices was 3.0(IQR: 2.0 to 4.0). The mean infarction lesion volume was $1.86 \pm 2$. $92 \mathrm{~cm}^{3}$. The number of patients with proximal infarction lesion was 20(62.5\%). 19(59.4\%) patients had significant leukoaraiosis (Fazekas grade $\geqq 2$ ).

\section{Comparison of patient characteristics between patients with and without HR-MRI identified plaques}

The plaque group had a higher percentage of diabetes mellitus than non-plaque group $(7[46.7 \%]$ versus $1[5.9 \%]$; $P=0.013)$. No other significant differences were found in terms of demographic characteristics, atherosclerotic risk factors, NIHSS and mRS between the two groups (Table 2). On the contrast to the non-plaque group, the plaque group had a significantly larger infarction lesion length $(1.95 \pm 0.86$ versus $1.38 \pm 0.55 \mathrm{~cm} ; P=0.031)$. Besides, the lesion volume was significantly larger in patients with plaque than in patients without plaque $(2.95 \pm 3.94$ versus $\left.0.90 \pm 0.94 \mathrm{~cm}^{3} ; P=0.027\right)$. Of the 32 patients, the median number of infarction lesion slices was 3.0. Patients were divided into two groups: patients with number of lesion slices $\geqq 3.0$ and patients with number of lesion slices <3.0. The plaque group had more patients with number of lesion slices $\geqq 3.0$ than non-plaque group with

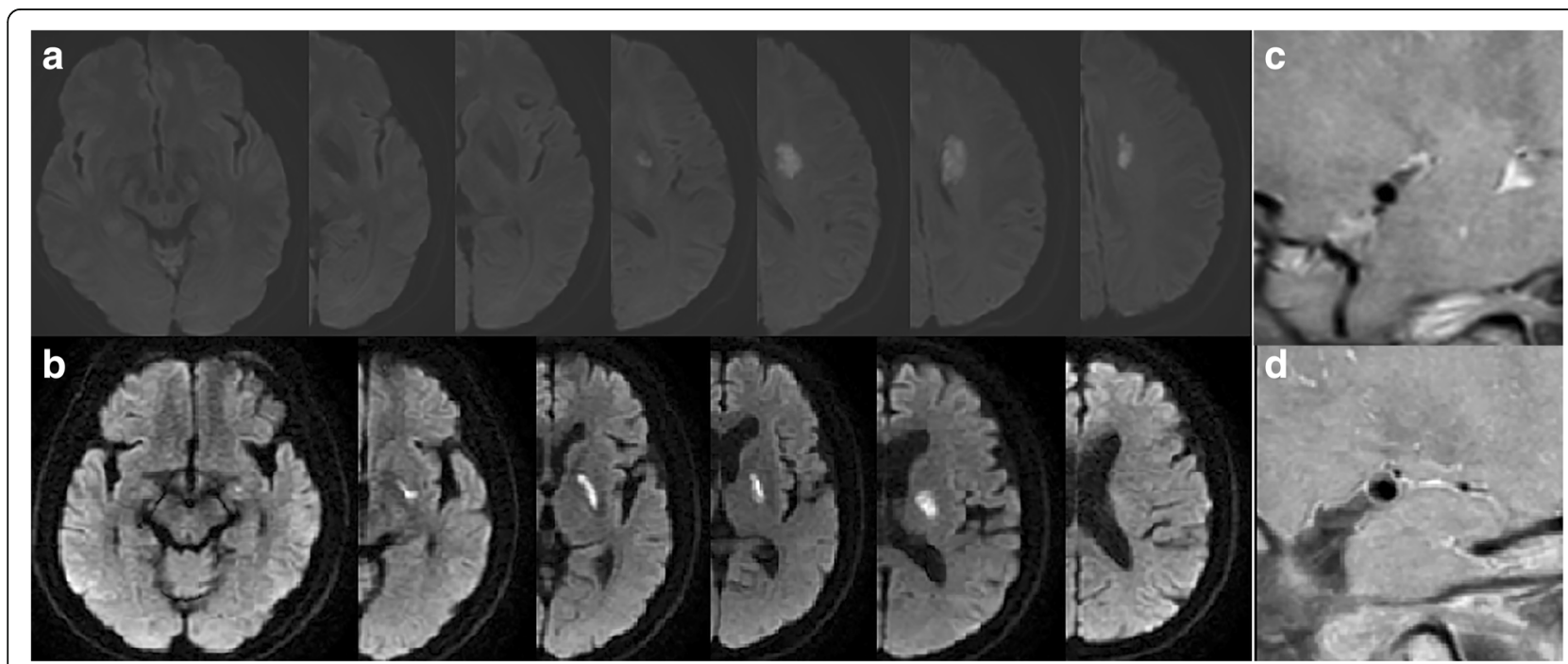

Fig. 1 Two types of single lenticulostriate artery territory infarction with no relevant MCA disease on MRA: (a) Distal single lenticulostriate artery territory infarction lesion (not extending to the basal surface of the MCA), (b) Proximal single lenticulostriate artery territory infarction lesion (extending to the basal surface of MCA), (c) HRMRI showed no plaque on MCA, (d) HRMRI showed plaque on MCA 
Table 1 VISTA images of all enrolled patients

\begin{tabular}{lllc}
\hline Non-Plaque & Plaque & Total \\
\hline $17(53.1 \%)$ & $15(46.9 \%)$ & 32 \\
& $\begin{array}{ll}\text { Location involving } \\
\text { dorsally upper part }\end{array}$ & Location not involving & \\
& dorsally upper part & \\
& $8(53.3 \%)$ & $7(46.7 \%)$ & \\
\hline
\end{tabular}

VISTA volumetric isotropic turbo spin echo acquisition

a borderline significance $(P=0.055)$. On the contrast to non-plaque group, the plaque group had a higher percentage of proximal lesions with a borderline significance $(P=0.055)$. There were no significant differences in terms of significant leukoaraiosis (Fazekas grade $\geqq 2$ ).

\section{Comparison of patient characteristics according to the location of MCA plaques}

We divided patients with HR-MRI identified MCA plaque into two groups: plaque location involving upper dorsally part and plaque location not involving upper dorsally part (Fig. 2). There were no significant differences in terms of imaging and clinical features (Table 3).

\section{Discussion}

It is usually difficult to distinguish BAD and non-branch atheromatous small vessel disease for practicing clinicians.
Microatheromas have been reported to be the most common underlying mechanism of symptomatic lacunar infarction in autopsy studies [10]. However, traditional angiography examinations, such as MRA, CTA and DSA, cannot identify BAD. Recent studies have reported HRMRI is an effective and directive tool for the evaluation of intracranial atherosclerotic plaques. In our study, we found that as many as $46.9 \%$ of acute lenticulostriate stroke patients with nonstenotic MCA on MRA demonstrated HR-MRI identified MCA plaques. This result is consistent with previous studies about MCA plaques in patients with lacunar infarction [11-13].

Previously identification of BAD is mainly based on the neuroimaging features on traditional brain MRI. However, different studies used different methods to define BAD. Yamamoto et al. defined BAD of the lenticulostriate stroke as infarcts more than $10 \mathrm{~mm}$ in diameter on axial slice and visible on 3 or more axial slices [3]. Jeong et al. defined BAD as lesions visible on 4 or more axial slices [14]. $\mathrm{Nah}$ et al. considered that infarction lesion involvement of the lowest portion of the basal ganglia was an indication of BAD [9]. A study from China, 312 patients with lacunar infarction who had normal MCA on MRA enrolled, suggested that infarct lesion size may be used as a method to identify BAD [15]. According to the studies mentioned

Table 2 Patient Characteristics According to the Presence of MCA Plaques

\begin{tabular}{|c|c|c|c|}
\hline Characteristics & $\begin{array}{l}\text { Plaque } \\
(n=15)\end{array}$ & $\begin{array}{l}\text { Non-Plaque } \\
(n=17)\end{array}$ & $P$ value \\
\hline \multicolumn{4}{|l|}{ Demographics } \\
\hline Age, years, mean $\pm S D$ & $63.47 \pm 10.00$ & $58.76 \pm 12.63$ & 0.257 \\
\hline Male, n(\%) & $10(66.7 \%)$ & $12(70.6 \%)$ & 0.811 \\
\hline \multicolumn{4}{|l|}{ Atherosclerotic risk factors } \\
\hline Hypertension, n (\%) & $12(80.0 \%)$ & $10(58.8 \%)$ & 0.197 \\
\hline Hyperlipidemia, n (\%) & $9(60.0 \%)$ & $11(64.7 \%)$ & 0.784 \\
\hline Diabetes mellitus, n (\%) & $7(46.7 \%)$ & $1(5.9 \%)$ & 0.013 \\
\hline Hyperhomocysteinemias, n (\%) & $2(13.3 \%)$ & $5(29.4 \%)$ & 0.402 \\
\hline Previous history of stroke, n (\%) & $2(13.3 \%)$ & $1(5.9 \%)$ & 0.589 \\
\hline Tobacco consumption, n (\%) & $3(20.0 \%)$ & $7(41.2 \%)$ & 0.197 \\
\hline \multicolumn{4}{|l|}{ Imaging features } \\
\hline Lesion length, cm (mean \pm SD) & $1.95 \pm 0.86$ & $1.38 \pm 0.55$ & 0.031 \\
\hline Number of lesion slices (median[IQR]) & $3.0(3.0-5.0)$ & $2.0(2.0-3.5)$ & 0.132 \\
\hline Number of lesion slices $\geqq 3, n$ (\%) & $12(80.0 \%)$ & $8(47.1 \%)$ & 0.055 \\
\hline Lesion volume, $\mathrm{cm}^{3}$ (mean $\pm \mathrm{SD}$ ) & $2.95 \pm 3.94$ & $0.90 \pm 0.94$ & 0.027 \\
\hline Proximal lesion, n (\%) & $12(80.0 \%)$ & $8(47.1 \%)$ & 0.055 \\
\hline Significant leukoaraiosis (Fazekas grade $\geqq 2$ ), n (\%) & $11(73.3 \%)$ & $8(47.1 \%)$ & 0.131 \\
\hline \multicolumn{4}{|l|}{ Severity of stroke } \\
\hline $\mathrm{NIHSS}$ at admission(median[IQR]) & $2.0(1.0-4.0)$ & $3.0(1.0-5.5)$ & 0.278 \\
\hline $\mathrm{NIHSS}$ at discharge(median[IQR]) & $1.0(0-3.0)$ & $2.0(1.0-4.0)$ & 0.628 \\
\hline $\mathrm{mRS} \geqq 2, \mathrm{n}(\%)$ & $4(26.7 \%)$ & $5(29.4 \%)$ & 1.000 \\
\hline
\end{tabular}

MCA middle cerebral artery, SD standard deviation, IQR interquartile range, NIHSS National Institutes of Health Stroke Scale, mRS modified Rankin Scale 


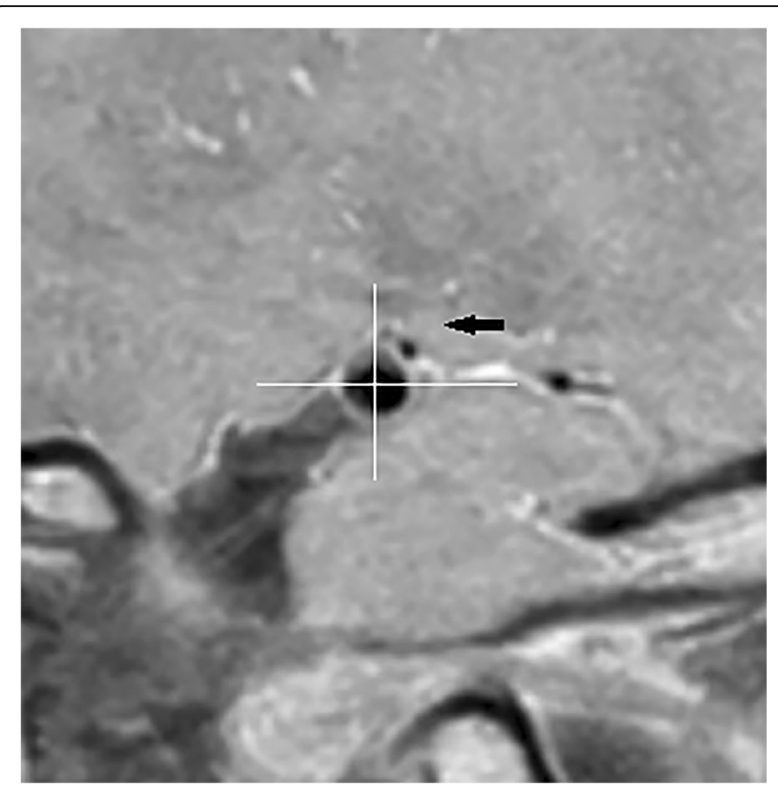

Fig. 2 Example of plaque location involving dorsally upper part. The black arrow showed the dorsally upper part

above, BAD tended to have larger infarcts and lesions were closer to the orifice of the perforating artery. However, these studies lacked directive atherosclerotic plaques evidences on HR-MRI. In our study, we showed that patients with HR-MRI identified plaque had larger infarction lesions $(P<0.05)$ and tended to present proximal lesions $(P=0.055)$ and $\geqq 3$ lesion slices $(P=0.055)$, which was consistent with the past studies about imaging features of $\mathrm{BAD}$. Lenticulostriate artery originate from the initial segment of MCA, BAD tend to lead large proximal infarction lesion we think it was caused by plaque within parent artery (MCA) blocking the lenticulostriate artery orifice (Fig. 3a). But we can't directly show the plaque and the orifice of perforator branches in HR-MRI due to the perivascular space or Virchow-Robin spaces. Kim et al. found that located plaque, and plaque length no related to large lenticulostriate infarction. And considered that it was due to the variation of branching pattern of perforators, which occlusion of a single lenticulostriate artery produced a variety of infarct sizes $[16,17]$. In fact, both of the two pathogenesis can lead to the large infarction, we need a better way to show the details of lenticulostriate artery from orifice to ending, which can be used to distinguish the two pathogenesis.

However, the frequencies of proximal infarction lesions were not significantly different between patients with plaque and without plaque. We speculated that this result was associated with some patients had microatheroma in the orifice of the lenticulostriate artery itself (Fig. 3). In this hypothesis, patients could have proximal infarction lesions, but without HR-MRI identified MCA plaque. Different from a group lenticulostriate arteries occluded by MCA atherosclerotic plaque, this group patients could present small infarction lesions. So we furtherly speculated that microatheroma in the orifice of the lenticulostriate artery itself was the possible pathogenesis of small proximal infarction lesion. Unfortunately, microatheroma in the orifice of the lenticulostriate artery itself could not identified directly by using HR-MRI. Besides, the branching patterns of MCA perforators vary greatly in terms of type $[18,19]$ and infarction size and location may also be related to these variations.

What's more, in our study 10 patients' infarction lengths were more than $20 \mathrm{~mm}$ which can defined as large lenticulostriate infarction, and of these 6 patients with HRMRI identified plaque. In the Trial of ORG 10172 in Acute Stroke Treatment (TOAST) criteria, lenticulostriate infarction which length less than $15 \mathrm{~mm}$ was belong to the smell-vessel occlusion subtype [20]. But several studies

Table 3 Patient Characteristics According to the Location of MCA Plaques

\begin{tabular}{|c|c|c|c|}
\hline & $\begin{array}{l}\text { Plaque location involving } \\
\text { dorsally upper part } \\
(n=8)\end{array}$ & $\begin{array}{l}\text { Plaque location not involving } \\
\text { dorsally upper part } \\
(n=7)\end{array}$ & $P$ Value \\
\hline \multicolumn{4}{|l|}{ Imaging features } \\
\hline Lesion length, $\mathrm{cm}$ (mean $\pm \mathrm{SD}$ ) & $1.86 \pm 0.81$ & $2.05 \pm 0.96$ & 0.686 \\
\hline Number of lesion slices (median[IQR]) & $3.0(3.0-4.75)$ & $3.0(2.0-5.0)$ & 0.779 \\
\hline Number of lesion slices $\geqq 3, n(\%)$ & $7(87.5 \%)$ & $5(71.4 \%)$ & 0.569 \\
\hline Lesion volume, $\mathrm{cm}^{3}$ (mean $\pm \mathrm{SD}$ ) & $3.47 \pm 5.10$ & $2.37 \pm 2.25$ & 0.955 \\
\hline Proximal lesion & $7(87.5 \%)$ & $5(71.4 \%)$ & 0.569 \\
\hline Significant leukoaraiosis (Fazekas grade $\geqq 2$ ), n (\%) & $6(75.0 \%)$ & $5(71.4 \%)$ & 1.000 \\
\hline \multicolumn{4}{|l|}{ Severity of stroke } \\
\hline NIHSS at admission & $2.0(1.0-3.75)$ & $1.0(0-7.0)$ & 0.867 \\
\hline NIHSS at discharge & $1.5(1.0-2.75)$ & $1.0(0-5.0)$ & 1.000 \\
\hline $\mathrm{mRS} \geqq 2$ & $2(25 \%)$ & $2(28.6 \%)$ & 1.000 \\
\hline
\end{tabular}




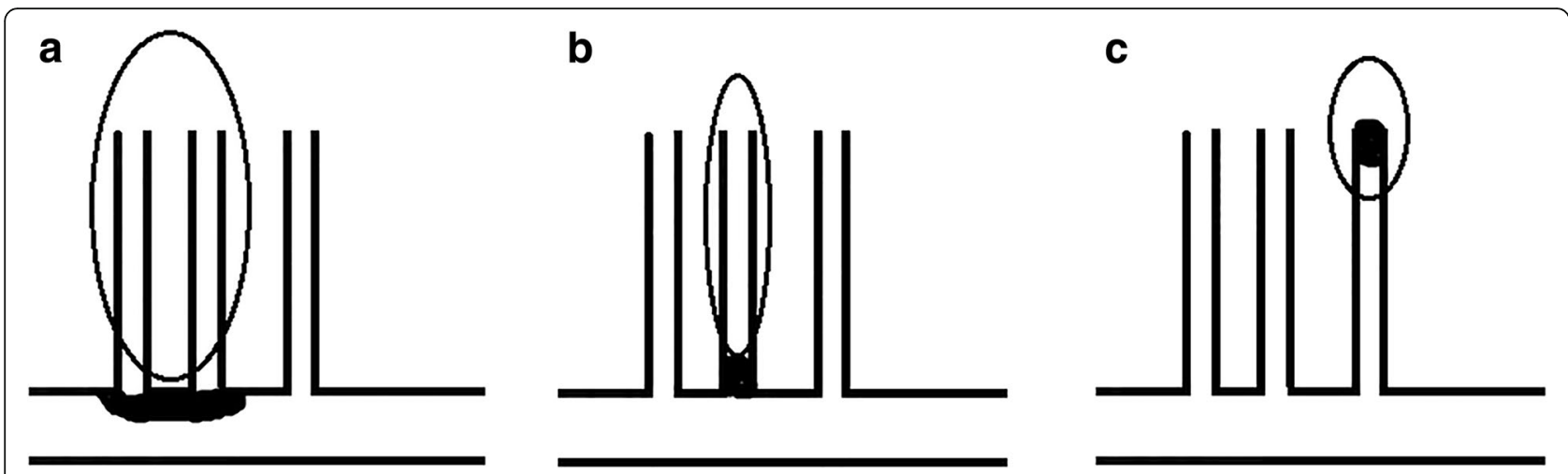

Fig. 3 Mechanisms of single lenticulostriate infarction: (a) Large proximal infarction lesion caused by plaque within parent artery(MCA) blocking the lenticulostriate artery orifice; (b) Proximal infarction lesion caused by microatheroma in the orifice of the lenticulostriate artery itself; (c) Distal lacunar infarction lesion caused by small vessel occlusion

found nearly half of patients with small vessel disease suffered infarction more than $15 \mathrm{~mm}$ [16], and small vessel disease can lead to an infarction with length even more than $20 \mathrm{~mm}$ because of occlusion of multiple perforating arterioles [21]. New ischemic stroke classifications included lesions of up to $20 \mathrm{~mm}$ in diameter [22] or even abandoned the rule of length of infarction [23, 24].

Theoretically, non-branch atheromatous small vessel disease is more strongly associated with hypertension and leukoaraiosis. Nevertheless, Yamamoto et al. found that there were no significant differences in prevalence of hypertension, diabetes mellitus, and hyperlipidemia between non-branch atheromatous small vessel disease and BAD [3]. In our study, we showed higher prevalence of diabetes mellitus in patients with MCA plaques than patients without MCA plaques. However, there were no differences in terms of other risk factors of atherosclerosis and significant leukoaraiosis between two groups.

Microanatomy studies suggested that most of penetrating arteries arose dorsally from the upper part of MCA wall [19]. We subdivided patients with MCA plaque into two groups: plaque location involving dorsally upper part and plaque location not involving dorsally upper part. However, there were no significant differences in terms of imaging features and severity of stroke between the two groups. We attributed this partly to the limited number of enrolled patients in our study and partly to the variations of branching patterns of MCA perforators [19].

\section{Conclusion}

Pathogenesis diagnosis of ischemic stroke is important for patient management. However, it is usually difficult to distinguish BAD from non-branch atheromatous small vessel disease for lenticulostriate infarction patients with nonstenotic MCA by using traditional imaging examinations. In our study, we demonstrated high frequency of MCA atheromatous plaques visualized on HR-MRI in patients with nonstenotic MCA on MRA. Patients with
HR-MRI identified plaque presented larger infarction lesions and more proximal lesions than patients without plaque, which were consistent with imaging features of BAD. HR-MRI was an important and effective tool for identifying stroke etiology, especially for patients with normal MCA by MRA. We strongly suggest that HR-MRI is particularly important for large proximal lenticulostriate infarction patients with nonstenotic MCA.

\section{Abbreviations \\ ACQ: Acquisition; BAD: branch atheromatous disease; CTA: computed tomography angiogram; DSA: digital subtraction angiography; DWl: diffusion weighted imaging; FOV: field of view; HR-MRl: high resolution magnetic resonance imaging; IQR: interquartile range; LAA: large artery atherosclerosis; MCA: middle cerebral artery; MRA: magnetic resonance angiography; mRS: Modified Rankin Scale; NIHSS: National Institutes of Health Stroke Scale; NSA: number of signal averages; REC: Reconstruction; SD: standard deviation; VISTA: volumetric isotropic turbo spin echo acquisition}

\section{Funding}

The work was supported by China-Japan Friendship Hospital Youth Science and Technology Excellence Project (No. 2014-QNYC-A-04), China-Japan Friendship Hospital Youth Science and Technology Project (No. 2015-2-QN-36), National Key Research and Development Program of China (No: 2016YFC0103003, 2016YFC0100105) and the Science and Technology Commission Foundation of Beijing (No: Z171100001017197) .

\section{Availability of data and materials}

The datasets used and/or analyzed during the current study are available from the corresponding author on reasonable request.

\section{Authors' contributions}

LLS, XJZ and ZJL designed this study. ZHL, WXT, WJY, and SL contributed to acquisition of data and analysis. LLS, FYC, LL and ZHL interpretation of data and manuscript draft. XBZ, XJZ and WXT revised manuscript. ZJL revise manuscript and gave final approval of the version to be published. All authors read and approved the final manuscript.

\section{Ethics approval and consent to participate}

This study approved by Institutional Ethics Committees of China-Japan Friendship Hospital,Beijing, China (No. 2017-65). The need for consent to participate was waived by the Institutional Ethics Committees of China-Japan Friendship Hospital because the study was an observational, retrospective study using a database from which the patients' identification information had been removed. Our study involved only the review of data contained in medical records did not require any involvement from the participants further than that of standard care. 


\section{Competing interests}

The authors declare that they have no competing interests.

\section{Publisher's Note}

Springer Nature remains neutral with regard to jurisdictional claims in published maps and institutional affiliations.

Received: 5 December 2017 Accepted: 18 April 2018

Published online: 25 April 2018

\section{References}

1. Kim D, Lee SH, Joon Kim B, Jung KH, Yu KH, Lee BC, Roh JK, for Korean stroke registry i. Secondary prevention by stroke subtype: a nationwide follow-up study in 46108 patients after acute ischaemic stroke. Eur Heart J. 2013;34(35):2760-7.

2. Damasio H. A computed tomographic guide to the identification of cerebral vascular territories. Arch Neurol. 1983;40(3):138-42.

3. Yamamoto Y, Ohara T, Hamanaka M, Hosomi A, Tamura A, Akiguchi I. Characteristics of intracranial branch atheromatous disease and its association with progressive motor deficits. J Neurol Sci. 2011;304(1-2):78-82

4. Caplan LR. Intracranial branch atheromatous disease: a neglected, understudied, and underused concept. Neurology. 1989;39(9):1246-50.

5. Zhu XJ, Wang W, Liu ZJ. High-resolution magnetic resonance Vessel Wall imaging for intracranial arterial stenosis. Chin Med J. 2016;129(11):1363-70.

6. Zhao DL, Deng G, Xie B, Ju S, Yang M, Chen XH, Teng GJ. High-resolution MRI of the vessel wall in patients with symptomatic atherosclerotic stenosis of the middle cerebral artery. J Clin Neurosci. 2015;22(4):700-4.

7. Ryu CW, Kwak HS, Jahng GH, Lee HN. High-resolution MRI of intracranial atherosclerotic disease. Neurointervention. 2014;9(1):9-20.

8. Inzitari D, Simoni M, Pracucci G, Poggesi A, Basile AM, Chabriat H, Erkinjuntti T, Fazekas F, Ferro JM, Hennerici M, et al. Risk of rapid global functional decline in elderly patients with severe cerebral age-related white matter changes: the LADIS study. Arch Intern Med. 2007;167(1):81-8.

9. Nah HW, Kang DW, Kwon SU, Kim JS. Diversity of single small subcortical infarctions according to infarct location and parent artery disease: analysis of indicators for small vessel disease and atherosclerosis. Stroke. 2010;41(12):2822-7.

10. Fisher CM. Capsular infarcts: the underlying vascular lesions. Arch Neurol. 1979;36(2):65-73.

11. Klein IF, Lavallee PC, Mazighi M, Schouman-Claeys E, Labreuche J, Amarenco P. Basilar artery atherosclerotic plaques in paramedian and lacunar pontine infarctions: a high-resolution MRI study. Stroke. 2010;41(7):1405-9.

12. Chung JW, Kim BJ, Sohn CH, Yoon BW, Lee SH. Branch atheromatous plaque: a major cause of lacunar infarction (high-resolution MRI study). Cerebrovasc Dis Extra. 2012;2(1):36-44.

13. Yoon Y, Lee DH, Kang DW, Kwon SU, Kim JS. Single subcortical infarction and atherosclerotic plaques in the middle cerebral artery: high-resolution magnetic resonance imaging findings. Stroke. 2013;44(9):2462-7.

14. Jeong HG, Kim BJ, Yang MH, Han MK, Bae HJ. Neuroimaging markers for early neurologic deterioration in single small subcortical infarction. Stroke. 2015;46(3):687-91.

15. Yang L, Qin W, Zhang X, Li Y, Gu H, Hu W. Infarct size may distinguish the pathogenesis of lacunar infarction of the middle cerebral artery territory. Med Sci Monit. 2016;22:211-8.

16. Cho AH, Kang DW, Kwon SU, Kim JS. Is 15 mm size criterion for lacunar infarction still valid? A study on strictly subcortical middle cerebral artery territory infarction using diffusion-weighted MRI. Cerebrovasc Dis. 2007;23(1):14-9.

17. Kim BJ, Lee DH, Kang D-W, Kwon SU, Kim JS. Branching patterns determine the size of single subcortical infarctions. Stroke. 2014;45(5):1485-7.

18. Marinkovic SV, Kovacevic MS, Marinkovic JM. Perforating branches of the middle cerebral artery. Microsurgical anatomy of their extracerebral segments. J Neurosurg. 1985;63(2):266-71.

19. Umansky F, Gomes FB, Dujovny M, Diaz FG, Ausman Jl, Mirchandani HG, Berman SK. The perforating branches of the middle cerebral artery. A microanatomical study. J Neurosurg. 1985;62(2):261-8.

20. Adams HP Jr, Bendixen BH, Kappelle LJ, Biller J, Love BB, Gordon DL, Marsh EE 3rd. Classification of subtype of acute ischemic stroke. Definitions for use in a multicenter clinical trial. TOAST. Trial of org 10172 in acute stroke treatment. Stroke. 1993;24(1):35-41.
21. Wardlaw JM, Smith C, Dichgans M. Mechanisms of sporadic cerebral small vessel disease: insights from neuroimaging. The Lancet Neurology. 2013; 12(5):483-97.

22. Ay $H$, Benner $T$, Murat Arsava E, Furie KL, Singhal AB, Jensen MB, Ayata C, Towfighi A, Smith EE, Chong JY, et al. A computerized algorithm for etiologic classification of ischemic stroke: the causative classification of stroke system. Stroke. 2007;38(11):2979-84.

23. Han SW, Kim SH, Lee JY, Chu CK, Yang JH, Shin HY, Nam HS, Lee BI, Heo JH. A new subtype classification of ischemic stroke based on treatment and etiologic mechanism. Eur Neurol. 2007;57(2):96-102.

24. Gao S, Wang Y, Xu A, Li Y, Wang D. Chinese ischemic stroke subclassification Front Neurol. $2011 ; 2: 6$.

\section{Ready to submit your research? Choose BMC and benefit from:}

- fast, convenient online submission

- thorough peer review by experienced researchers in your field

- rapid publication on acceptance

- support for research data, including large and complex data types

- gold Open Access which fosters wider collaboration and increased citations

- maximum visibility for your research: over $100 \mathrm{M}$ website views per year

At BMC, research is always in progress.

Learn more biomedcentral.com/submissions 